\title{
A slight extension of Euler's Theorem on Homogeneous Functions.
}

\author{
By W. E. Philip, M.A.
}

Euler's Theorem may be looked upon as the result of a certain operator acting on a special kind of function. This function may depend on any number of variables, but for convenience it is usual to consider three, viz., $x, y, z$. A function is homogeneous in $x, y, z$ and of the $n$th degree if it can be put into the form

$$
x " f\left(\frac{y}{x}, \frac{z}{x}\right)
$$

If we adopt for conciseness the following notation, viz,

$$
\begin{aligned}
& \Delta_{1}=x \frac{d}{d x}+y \frac{d}{d y}+z \frac{d}{d z}=x d_{1}+y d_{2}+z d_{3} \quad \text { say } \\
& \Delta_{2}=\left(x d_{1}+y d_{2}+z d_{3}\right)^{2}
\end{aligned}
$$

and generally $\Delta_{p}=\left(x d_{1}+y d_{2}+z d_{3}\right)^{p}$ where the multinomial function is to be expanded and then interpreted as an operator, we nuay state Euler's theorem thus

$$
\Delta_{p} u=n(n-1)(n-2) \ldots(n-p+1) u,
$$

where $u$ is a homogeneous function of $x, y, z$ of degree $u$.

In this note we wish to express the result of the same operator acting on any function of $u$, say $\mathrm{U} \equiv \mathrm{F}(u)$.

Now

$$
\begin{aligned}
& \begin{aligned}
\Delta_{1} \mathrm{U}=\left(x d_{1}+y d_{2}+z d_{3}\right) \mathrm{F}(u)=\mathrm{F}^{\prime}(u)\left\{x \frac{\partial u}{\partial x}\right. & \left.+y \frac{\partial u}{\partial y}+z \frac{\partial u}{\partial z}\right\}=n u \mathrm{~F}^{\prime}(u) \\
& =n u \frac{d}{d u} \mathrm{~F}(u)=\mathrm{F}_{1}(u), \text { say. }
\end{aligned} \\
& \Delta_{1}{ }^{2} \mathrm{U}=\Delta_{1} \mathrm{~F}_{1}(u)=n u \frac{d}{d u} \mathrm{~F}_{1}(u)=\left(n u \frac{d}{d u}\right)^{2} \mathrm{~F}(u),
\end{aligned}
$$

and generally

$$
\Delta_{1}^{m} \mathrm{U}=\left(n u \frac{d}{d u}\right)^{m} \mathrm{~F}(u)
$$


If we make the substitution $u=e^{n \theta}$, this gives

$$
\Delta_{1}^{n l} \mathbf{F}(u)=\left(\frac{d}{d \theta}\right)^{m} \mathbf{F}\left(e^{n \theta}\right)=\delta^{m l} \mathbf{F}\left(e^{n \theta}\right), \quad \text { where } \delta \equiv \frac{d}{d \theta} .
$$

Hence it follows that any rational integral function of $\Delta_{1}$ may be expressed in terms of $\delta$.

Thus

$$
\phi\left(\Delta_{1}\right) \mathbf{F}(u)=\phi(\delta) \mathbf{F}\left(e^{n \theta}\right) .
$$

Now let

$$
\Delta_{\mu} \mathrm{U} \text { be denoted by } \mathrm{U}_{\mu} \text {. }
$$

We have

$$
\Delta_{1} \mathrm{U}_{p}=\mathrm{U}_{p+1}+p \mathrm{U}_{p} \text {. }
$$

For we have first to apply the operator $x d_{1}+y d_{2}+z d_{3}$ to the various powers of $d_{3}, d_{2}, d_{3}$ in the expression $\Delta_{p}$, and this produces $\mathrm{U}_{p^{\prime+1}}$; then to the various powers of $x, y, z$, and this, by Euler's theorem, gives $p \mathrm{U}_{,}$.

Thus

$$
\begin{aligned}
& \mathrm{U}_{\mu+1}=\left(\Delta_{1}-\nu\right) \mathrm{U}_{\mu} \\
& \mathrm{U}_{p^{\prime}}=\left(\Delta_{1}-\overline{p-1}\right) \mathrm{U}_{p^{\prime-1}} \\
& \mathrm{U}_{1}=\Delta_{\mathrm{l}} \mathrm{U} \\
& \therefore \quad \mathrm{U}_{p+1}=\left(\Delta_{1}-p\right)\left(\Delta_{1}-\overline{p-1}\right) \ldots \Delta_{1} \mathrm{U} \\
& =\phi\left(\Delta_{1}\right) \mathbf{F}(u)=\phi(\delta) \mathbf{F}\left(e^{n \theta}\right) \\
& =\delta(\delta-1) \ldots(\delta-p) \mathbf{F}\left(e^{\lambda \theta \theta}\right) .
\end{aligned}
$$$$
\text { Again }
$$$$
\text { and }
$$

Now put $\lambda=e^{\theta}$ and we get by a well-known theorem

This is the result desired.

$$
U^{p+1}=\lambda^{\mu+1} \frac{d^{p+1}}{d \lambda^{\mu+1}} \mathrm{~F}\left(\lambda^{n}\right)
$$

Suppose, for example, $\mathrm{F}(u)=u$

then

$$
\begin{gathered}
\mathrm{U}_{p}=\lambda^{\prime} \frac{d^{\prime \prime}}{d \lambda^{\prime \prime}}\left(\lambda^{n}\right)=n(u-1) \ldots(n-p+1) \lambda^{n} \\
=n(n-1)(u-p+1) u, \quad \text { Euler's result. } \\
\mathrm{F}(u) \equiv \log u=\log \lambda^{n}=n \log \lambda \\
\mathrm{U}_{\nu^{\prime}}=(-1)^{p+1}(p-1) !
\end{gathered}
$$

Again take $\quad \mathrm{F}(u) \equiv \log u=\log \lambda^{n}=n \log \lambda$ result

The case where $u$ is homogeneous and of the first degree gives the

$$
\mathrm{U}_{p}=\lambda^{\prime \prime} \frac{d^{\prime \prime}}{d \lambda^{p}} \mathrm{~F}(\lambda)=u^{\prime \prime} \mathbf{F}^{(\mu)}(u)
$$

Other examples might be written down. 